\title{
Le long cheminement en France de la réflexion sur la fin de vie
}

\author{
Jean Martin \\ Dr méd., membre de la rédaction
}

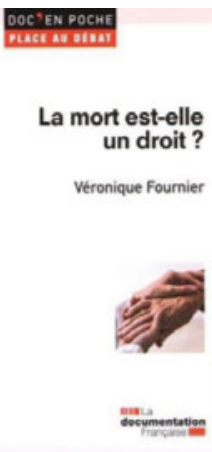

Véronique Fournier La mort est-elle un droit?

Paris: La Documentation Française (Série «Place au débat») 2016. 164 pages, 7.90 EUR.
Le Dr Véronique Fournier a créé le Centre d'éthique de l'hôpital Cochin, à Paris, dont elle a rendu compte de l'expérience dans des publications substantielles [1], et elle préside depuis 2016 le Centre national des soins palliatifs et de la fin de vie. Dans son dernier ouvrage, elle décrit le cheminement en France, depuis la fin du $\mathrm{XX}^{\mathrm{e}}$ siècle, des idées et démarches à propos de fin de vie; dans les milieux de l'éthique, le corps médical, le monde politique, jusqu'à la révision récente, dite Claeys Leonetti, de la loi Leonetti de 2005 [2]. Révision qui, quant aux points essentiels, dispose que les directives anticipées du patient s'imposent aux médecins et soignants et ouvre, sous conditions, un droit du patient en fin de vie à bénéficier d'une sédation profonde et continue jusqu'au décès - sédation terminale.

L'auteure reste au plus près de la réalité clinique, rappelant que ces préoccupations se sont aiguisées depuis les années 1970 avec les avancées de la réanimation médicale. Elle considère les questions éthiques et philosophiques de même que le processus politique - tout en prenant du recul par rapport aux combats très marqués d'idéologie du passé récent. Elle souligne comment accorder plus de place au respect de l'opinion de l'autre, et faire un effort concerté de tolérance au meilleur sens du terme, «la voie du dialogue pour le vivre ensemble», serait susceptible d'apaiser une scène politico-sociétale qui a pris des airs de guerres de religion (ce vocable est de J.M.).

En effet, ne devrait-il pas être admissible, et même salué, au pays des droits de l'homme, que des individus majeurs soient en mesure de prendre des décisions concernant leur mort comme ils en prennent à propos de leur vie? Etant entendu que, en aucune manière, la décision de l'un (cas échéant, demander une assistance à mourir) ne saurait être au détriment de la volonté différente de l'autre. Fournier consacre un de ses cinq chapitres à décrire comment cela est mis en œuvre dans un nombre croissant de pays, dont la Suisse. «L'une des difficultés du sujet est d'être aux confins de l'individuel et du collectif. Rien de plus respectable que d'avoir sa propre opinion sur la façon dont on aimerait pouvoir mourir. Et pourtant, arrivé à cette extrémité, on a en général besoin d'autrui, donc du collectif, selon la façon dont on conçoit ce que l'on doit à l'autre au nom du vivre ensemble.»

Noter que la question divise aussi la communauté des soins palliatifs - pas seulement en France: une partie d'entre elle s'oppose inconditionnellement à toute mesure où elle perçoit une "pente glissante» vers l'euthanasie, alors que d'autres voient comment soins palliatifs classiques et, par exemple, la sédation terminale maintenant admise peuvent véritablement être complémentaires, devenant des "soins palliatifs intégraux", et contribuer à une meilleure prise en charge, pour patients et proches.

Fournier relève que la résistance est jusqu'ici souvent plus forte dans les milieux soignants, pour des motifs qu'on peut comprendre - la crainte d'être de plus en plus souvent témoins de fins de vie «décidées" («commercer quotidiennement avec la mort»). Aussi, les empoignades philosophiques n'ont rien fait pour une compréhension plus posée des réalités pratiques. «On ne peut pas laisser des situations pourrir au nom du dogme de la mort naturelle", il importe de "travailler loin de tout dogmatisme». Ceci alors que les études d'opinions montrent qu'une majorité de Français se disent ouverts à un droit de décider de sa propre mort.

Difficile de savoir comment les choses vont évoluer. Il n'est pas exclu que «le socle de la loi Claeys Leonetti soit trop instable, puisque fondé sur la ligne de crête d'un improbable compromis, susceptible de ne contenter ni les partisans de l'ouverture d'un droit à mourir ni ses opposants.» Devant l'histoire récente de désaccords, l'auteure n'ose guère espérer pour bientôt l'avènement d'un véritable consensus. Regrettable - mais le pire n'est jamais certain.

\section{Références}

1 Martin J. Fin de vie, soins palliatifs, euthanasie - Où sont les démarcations qui valent? (à propos de "Puisqu'il faut bien mourir», de Véronique Fournier). Bull Méd Suisses. 2016. 97:267-9.

2 Martin J. Fin de vie, mort, médecine (à propos de l'ouvrage "C'est ainsi que les hommes meurent», de Jean Leonetti). Bull Méd Suisses. 2015.96:1396-7. 